\title{
Fermentation and Metabolic Pathway Optimization to De Novo Synthesize (2S)- Naringenin in Escherichia coli
}

\author{
Shenghu Zhou ${ }^{1,2+*}$, Tingting $\mathrm{Hao}^{1,2+}$, and Jingwen $\mathrm{Zhou}^{1,2 *}$ \\ 'National Engineering Laboratory for Cereal Fermentation Technology, Jiangnan University, Wuxi, Jiangsu 214122, \\ P.R. China \\ ${ }^{2}$ Jiangsu Provincial Research Center for Bioactive Product Processing Technology, Jiangnan University, Wuxi, \\ Jiangsu 214122, P.R. China
}

Flavonoids have diverse biological functions in human health. All flavonoids contain a common 2phenyl chromone structure (C6-C3-C6) as a scaffold. Hence, in using such a scaffold, plenty of highvalue-added flavonoids can be synthesized by chemical or biological catalyzation approaches. (2S)Naringenin is one of the most commonly used flavonoid scaffolds. However, biosynthesizing (2S)naringenin has been restricted not only by low production but also by the expensive precursors and inducers that are used. Herein, we established an induction-free system to de novo biosynthesize (2S)-naringenin in Escherichia coli. The tyrosine synthesis pathway was enhanced by overexpressing feedback inhibition-resistant genes ( $a r o G^{\mathrm{fbr}}$ and $t y r A^{\mathrm{fbr}}$ ) and knocking out a repressor gene (tyrR). After optimizing the fermentation medium and conditions, we found that glycerol, glucose, fatty acids, potassium acetate, temperature, and initial pH are important for producing (2S)-naringenin. Using the optimum fermentation medium and conditions, our best strain, Nar-17LM1, could produce $588 \mathrm{mg} / \mathrm{l}(2 \mathrm{~S})$-naringenin from glucose in a 5-L bioreactor, the highest titer reported to date in $E$. coli.

Keywords: L-tyrosine, $p$-coumaric acid, dynamic regulation, flavonoids, temperature-shift

Received: August 2, 2020 Accepted: August 19, 2020

First published online: August 21, 2020

*Corresponding authors S.Zhou

Phone: +86-510-85329031

Fax: +86-510-85918309

E-mail: zhoush@jiangnan.edu.cn J.Zhou

E-mail: zhoujw1982@jiangnan. edu.cn

'These authors contributed equally to this work.

pISSN 1017-7825

elSSN 1738-8872

Copyright(C) 2020 by

The Korean Society for

Microbiology and

Biotechnology

\section{Introduction}

Flavonoids are value-added nutritional chemicals that have diverse biological functions; they are antibacterial, anti-atherosclerotic, anti-carcinogenic, and they protect the liver $[1,2]$. Under the current industrial production scale, flavonoids are extracted mainly from plant tissues $[3,4]$. However, the low content in plant tissues results in a low titer and a high price for flavonoids that cannot satisfy the demands of the market [5]. Furthermore, the harmful solvents and extreme conditions needed in the extraction process also hinder the application of plant extraction [3]. Hence, it is necessary to develop a flavonoid production process that is environmentally friendly and has high efficiency.

Although there are thousands of flavonoids with significantly different structures, all of them contain a common 2-phenyl chromone structure (C6-C3-C6) as a scaffold [6]. Hence, any desired flavonoid could be synthesized from a flavonoid scaffold by either enzymatic or chemical catalyzation methods. In this regard, the achievement of large-scale production by a microbial fermentation approach using flavonoid scaffolds is important for green production. (2S)-Naringenin is one of the most common flavonoid scaffolds and has many nutritional and pharmaceutical functions in human health $[1,7,8] . p$-Coumaric acid and tyrosine are important precursors of (2S)-naringenin. Therefore, some researchers have used them to produce (2S)-naringenin. Using tyrosine as a substrate, Wu et al. fine-tuned the metabolic flux of the malonyl-CoA synthesis pathway through antisense RNA [9] and CRISPR interference [10] approaches, obtaining $391 \mathrm{mg} / \mathrm{l}$ and $421.6 \mathrm{mg} / \mathrm{l}$ of $(2 S)$ naringenin in Escherichia coli, respectively. In Saccharomyces cerevisiae, Lyu et al. obtained $\sim 90 \mathrm{mg} / \mathrm{l}$ of naringenin from tyrosine after optimizing the malonyl-CoA and naringenin synthesis pathway [11]. Compared with tyrosine, $p$-coumaric acid as a substrate could significantly improve the titer of (2S)-naringenin. In recent reports, Gao et al. systemically optimized the synthesis pathway of $(2 S)$-naringenin in S. cerevisiae, resulting in titers of $648.63 \mathrm{mg} / \mathrm{l}$ and $1.21 \mathrm{~g} / \mathrm{l}$ by using $p$-coumaric acid as a substrate $[12,13]$. These researchers have shown that engineered microbes are capable of producing flavonoids on a large scale.

However, the added tyrosine and $p$-coumaric acid increase the cost of fermentation. Hence, cheaper carbon sources are preferred in the fermentation process. To synthesize (2S)-naringenin from glucose, Wu et al. overexpressed 3-deoxy-D-arabinoheptulosonate-7-phosphate (DAHP) synthase (aroG ${ }^{\mathrm{fbr}}$ ) and chorismate mutase/prephenate dehydrogenase $\left(t y r A^{\text {fbr }}\right)$ in $E$. coli. This released the feedback inhibition by tyrosine to finally yield $100.64 \mathrm{mg} / \mathrm{l}(2 S)$-naringenin [14]. Furthermore, Raman et al. obtained $61 \mathrm{mg} / \mathrm{l}$ of $(2 S)$-naringenin from 

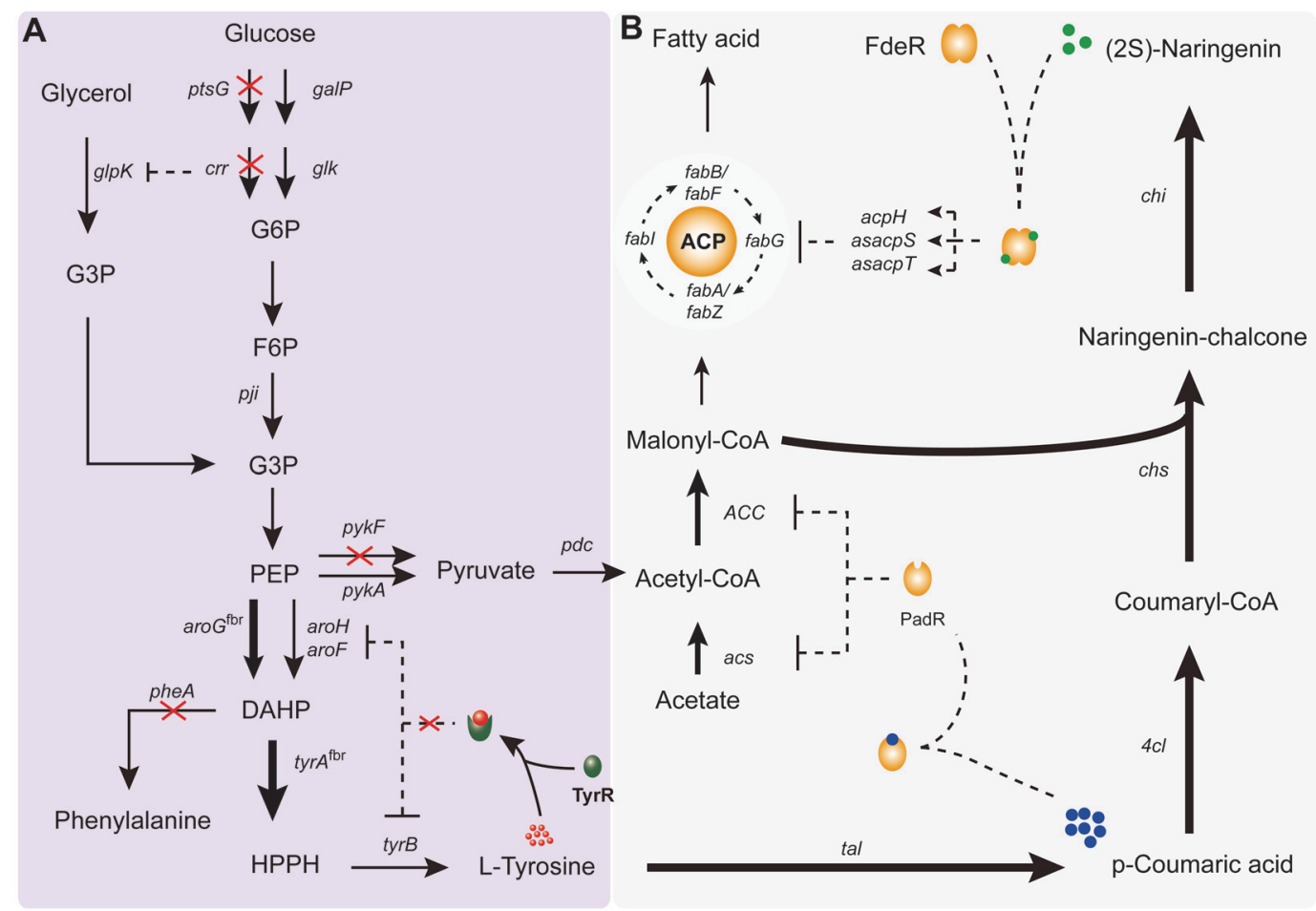

Fig. 1. The de novo biosynthesis pathway for (2S)-naringenin. (A) Metabolic engineering to enhance the metabolic flux from glucose to L-tyrosine. The red crosses represent genes that were deleted. The bold arrows represent genes that were overexpressed. The thin arrows represent genes that were natively expressed. (B) The dynamic regulation network of Mut-17, which was constructed in our previous study [19]. (2S)-Naringenin synthesis pathway was constitutively overexpressed [7]. Hence, (2S)-naringenin could accumulate with cell growth. The accumulated (2S)-naringenin activates activator FdeR [37] to repress the fatty acid synthesis pathway to reduce the consumption of malonyl-CoA. Meanwhile, the repressor PadR [38] can be inactivated when $p$-coumaric acid accumulates, resulting in the expression of acetyl-CoA synthase (acs) and acetyl-CoA carboxylase $(A C C)$ to enhance the biosynthesis of malonyl-CoA. Compound annotation: glyceraldehyde 3-phosphate (G3P); phosphoenolpyruvate (PEP); glucose-6-phosphate (G6P); fructose-6-phosphate (F6P); 4 hydroxyphenylpyruvate (HPPH); Gene or enzyme annotation: glycerol kinase $(g l p K)$; pyruvate kinase I/II ( $p y k F / p y k A)$; tyrosine repressor (TyrR); acyl carrier protein (ACP); tyrosine aminotransferase (tyrB); DAHP synthase (aroG/aroH/aroF); chorismate mutase/prephenate dehydratase (pheA); glucose-specific IIACB component ( $p t s G / c r r)$; galactose permease (galP); glucokinase (glk); phosphodiesterase $(a c p H)$; anti-sense RNA of holo-ACP synthase (asacpS) and holo-ACP synthase 2 (asacpT); tyrosine ammonia-lyase (tal), 4-coumarate: CoA ligase ( $4 c l$ ), chalcone synthase (chs), chalcone isomerase (chi).

glucose in E. coli by a biosensor-based directed evolution strategy [15]. According to recent reports, Yarrowia lipolytica generated a higher (2S)-naringenin titer than E. coli. Lv et al. optimized the biosynthetic pathway of (2S)naringenin by multi-module strategy and obtained a $252.4 \mathrm{mg} / \mathrm{l}$ titer from glucose [16]. Wei et al. obtained a 715.3 $\mathrm{mg} / \mathrm{l}$ titer in Y. lipolytica by using a xylose-responsive biosensor to control expression of the (2S)-naringenin synthesis pathway [17]. In this process, xylose simultaneously acted as a substrate and inducer. Recently, Palmer $e t$ al. broke this record by applying a $\beta$-oxidation-related strategy in Y. lipolytica. They generated $898 \mathrm{mg} / \mathrm{l}$ of $(2 S)$ naringenin from glucose, the highest titer reported to date in any host [18].

According to current reports, the titer of (2S)-naringenin from E. coli as host has usually been far lower than from either S. cerevisiae or Y. lipolytica. However, the various superior properties of E. coli, such as its easy engineering and fast growth, make it one of the best hosts for $(2 S)$-naringenin production. Hence, it is important to further improve the titer of (2S)-naringenin through de novo biosynthesis. In this study, we established an induction-free system in our previously constructed dynamic regulated $E$. coli strain, which can achieve automatic balancing of (2S)-naringenin production, p-coumaric acid accumulation, and cell growth (Figs. 1A and 1B) [19]. To do this, we first optimized the fermentation conditions ( $\mathrm{pH}$ and temperature) and medium contents (carbon sources, nitrogen source, and $\mathrm{MnCl}_{2}$ ). Then, we optimized the tyrosine synthesis pathway for de novo biosynthesis of (2S)-naringenin. The optimum strain produced $588 \mathrm{mg} / \mathrm{l}(2 S)$-naringenin from glucose in a 5 - $\mathrm{L}$ bioreactor. This is the highest titer reported to date in E. coli.

\section{Materials and Methods}

Strains, Medium, and Culture Conditions

E. coli JM109 and E. coli BL21 (DE3) were used as hosts for plasmid construction and pathway expression, respectively. Luria-Bertani broth (LB) medium was used to enrich cells for plasmid construction or fermentation 
seed culture. MOPS minimal medium [20] (supplemented with $5 \mathrm{~g} / \mathrm{l} \mathrm{D}$-glucose and $4 \mathrm{~g} / \mathrm{l} \mathrm{NH}_{4} \mathrm{Cl}$ ) was used as the initial medium for fermentation optimization. The L-tyrosine was dissolved in sodium hydroxide solution to a final concentration of $300 \mathrm{mM}$. Then, $1 \%$ of L-tyrosine solution was added in the medium to obtain the $3 \mathrm{mM}$ final concentration. In the fermentation optimization process, strains were cultured at $30^{\circ} \mathrm{C}$ and $220 \mathrm{rpm}$. Shake flask fermentation was performed at $220 \mathrm{rpm}$ with $3 \%$ of seed cultures under optimum fermentation conditions and medium. Ampicillin $(100 \mu \mathrm{g} / \mathrm{ml})$, streptomycin $(50 \mu \mathrm{g} / \mathrm{ml})$, chloramphenicol $(34 \mu \mathrm{g} / \mathrm{ml})$, and kanamycin $(50 \mu \mathrm{g} / \mathrm{ml})$ were added to the media as required. The details of the strains and plasmids used in this study are listed in Table 1.

Table 1. Strains and plasmids used in this study.

\begin{tabular}{|c|c|c|}
\hline Strains and plasmids & Properties & Sources \\
\hline \multicolumn{3}{|l|}{ Strains } \\
\hline E. coli JM109 & Wild type & This study \\
\hline E. coli BL21 (DE3) & Wild type & This study \\
\hline Mut-17 & 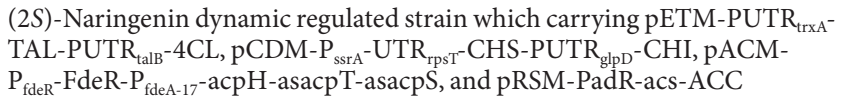 & [19] \\
\hline Nar-17LL & 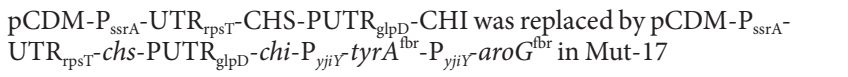 & This study \\
\hline Nar-17HL & 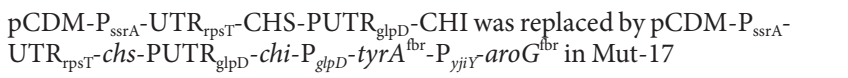 & This study \\
\hline Nar-17HM & 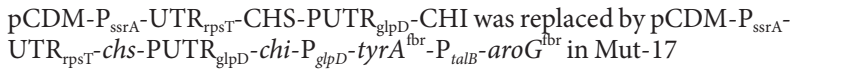 & This study \\
\hline Nar-17LM & 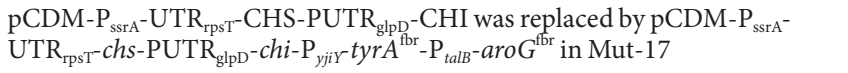 & This study \\
\hline Nar-17LM1 & tyrR was knockout in Nar-17LM & This study \\
\hline Nar-17LM2 & tyrR, pst $G$, and $c r r$ were knockout in Nar-17LM & This study \\
\hline Nar-17LM3 & tyrR, pst $G, c r r$, and $p h e A$ were knockout in Nar-17LM & This study \\
\hline Nar-17LM4 & tyrR, pstG, crr, phe A, and $p y k F$ were knockout in Nar-17LM & This study \\
\hline
\end{tabular}

Plasmids

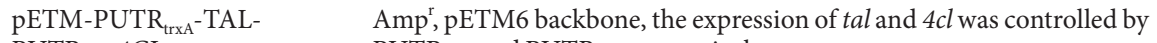

$\mathrm{PUTR}_{\mathrm{talB}}-4 \mathrm{CL}$

PUTR $_{\text {trxA }}$ and PUTR talB $_{\text {, }}$ respectively.

pCDM- $\mathrm{P}_{\text {ssrA }}-\mathrm{UTR}_{\mathrm{rpsT}}-\mathrm{CHS}-\quad$ Strr ${ }^{\mathrm{r}}$, pCDM4 backbone, the expression of chi and chs was controlled by $\mathrm{P}_{\text {ssrA }^{-}}$

PUTR $_{\mathrm{glpD}}-\mathrm{CHI}$

$\mathrm{UTR}_{\mathrm{rpsT}}$ and PUTR $\mathrm{glpD}$, respectively.

pACM- $\mathrm{P}_{\text {fdeR }}$-FdeR-P $\mathrm{P}_{\text {fdeA- } 17^{-}}$

Cm ${ }^{\mathrm{r}}$, pACM4 backbone, $\mathrm{P}_{\mathrm{fdeR}}$ and $\mathrm{P}_{\mathrm{fdeA}-17}$ controlled the expression of $f d e R$ and $a c p H$, respectively. $\mathrm{P}_{\mathrm{fdeA}}$ controlled the expression of asacpT and asacpS.

pRSM-PadR-acs-ACC

$\mathrm{Kan}^{\mathrm{r}}$, pRSM3 backbone, $\mathrm{P}_{\mathrm{cspA}}$ and $\mathrm{P}_{\text {padC }}$ controlled the expression of padR and acs, $A C C$, respectively.

pMD-tyrA $A^{\mathrm{fbr}}-\operatorname{aro} G^{\mathrm{fb}}$

pMD- $\mathrm{P}_{y j i Y}$ tyr $A^{\mathrm{fbr}}$-aro $G^{\mathrm{fbr}}$

Amp ${ }^{\mathrm{r}}$, T-vector pMDTM19 (Simple) carrying $t y r A^{\mathrm{fbr}}$ and $\operatorname{aro} G^{\mathrm{fbr}}$

Amp ${ }^{\mathrm{r}}, \mathrm{pMD}-t y r A^{\mathrm{fbr}}$-aro $G^{\mathrm{fbr}}$ backbone, $\mathrm{P}_{y j i \mathrm{Y}}$ controlled the expression of $t y r A^{\mathrm{fbr}}$

pMD- $\mathrm{P}_{g l p D}-t y r A^{\mathrm{fbr}}-\operatorname{aro} G^{\mathrm{fbr}}$

Amp ${ }^{\mathrm{r}}$, pMD-tyr $A^{\mathrm{fbr}}$-aro $G^{\mathrm{fbr}}$ backbone, $\mathrm{P}_{g l p D}$ controlled the expression of tyra $A^{\text {fbr }}$

pMD- $\mathrm{P}_{y j i Y}$ tyr $A^{\mathrm{fbr}}-\mathrm{P}_{\text {talB }}-$ aro $G^{\mathrm{fbr}} \quad$ Amp ${ }^{\mathrm{r}}, \mathrm{pMD}-\mathrm{P}_{y j i Y} t y r A^{\mathrm{fbr}}$-aro $G^{\mathrm{fbr}}$ backbone, $\mathrm{P}_{\text {talB }}$ controlled the expression of $\operatorname{aroG}^{\text {fbr }}$

pMD- $\mathrm{P}_{y j i Y} t y r A^{\mathrm{fbr}}-\mathrm{P}_{y j i Y}$ aro $G^{\mathrm{fbr}}$

Amp ${ }^{\mathrm{r}}, \mathrm{pMD}-\mathrm{P}_{y j i Y} t y r A^{\mathrm{fbr}}$-aro $G^{\mathrm{fbr}}$ backbone, $\mathrm{P}_{y j i Y}$ controlled the expression of $\operatorname{aroG}^{\mathrm{b}}$

$\mathrm{pMD}-\mathrm{P}_{g l p D}-t y r A^{\mathrm{fbr}}-\mathrm{P}_{y j i Y}-\operatorname{aro} G^{\mathrm{fbr}}$

Amp ${ }^{\mathrm{r}}, \mathrm{pMD}-\mathrm{P}_{g l p D^{-}}$tyr $A^{\mathrm{fbr}}$-aro $G^{\mathrm{fbr}}$ backbone, $\mathrm{P}_{y j i Y}$ controlled the expression of $\operatorname{aroG}^{\mathrm{fbr}}$

pMD- $\mathrm{P}_{g l p D}-t y r A^{\mathrm{fbr}}-\mathrm{P}_{\text {talB }}$-aro $G^{\mathrm{fbr}}$

$A m p^{\mathrm{r}}$, pMD- $\mathrm{P}_{g l p D^{-}} t y r A^{\mathrm{fbr}}$-aro $G^{\mathrm{fbr}}$ backbone, $\mathrm{P}_{\text {talB }}$ controlled the expression of $\operatorname{aroG}^{\mathrm{fbr}}$

pCDM- $\mathrm{P}_{\mathrm{ssrA}^{-}}-\mathrm{UTR}_{\mathrm{rpsT}}-$ chs-

Str ${ }^{\mathrm{r}}$, pCDM- $\mathrm{P}_{\text {ssra }}-\mathrm{UTR}_{\mathrm{rpsT}}-\mathrm{CHS}-\mathrm{PUTR}_{\mathrm{glpD}}-\mathrm{CHI}$ backbone, $\mathrm{P}_{y j \mathrm{I} Y}$ and $\mathrm{P}_{\text {talB }}$

$\mathrm{PUTR}_{\mathrm{glpD}}-$ chi- $\mathrm{P}_{y j i Y^{\prime}}$ tyr $A^{\mathrm{fbr}}-\mathrm{P}_{\text {talB }}{ }^{-}$

controlled the expression of $t y r A^{\mathrm{fbr}}$ and $\operatorname{aro}^{\mathrm{fbr}}$, respectively.

$\operatorname{aro} G^{\mathrm{fbr}}$

Str $^{\mathrm{r}}, \mathrm{pCDM}-\mathrm{P}_{\text {ssra }}-\mathrm{UTR}_{\mathrm{rpsT}}-\mathrm{CHS}-\mathrm{PUTR}_{\mathrm{glpD}}-\mathrm{CHI}$ backbone, $\mathrm{P}_{y j i Y}$ and $\mathrm{P}_{y j i Y}$
controlled the expression of $t y r A^{\mathrm{fbr}}$ and aro $G^{\mathrm{fbr}}$, respectively.

pCDM- $\mathrm{PsrA}_{\mathrm{SPT}^{-}}-\mathrm{UTR}_{\mathrm{rpsT}}-$ chs-

$\operatorname{PUTR}_{\operatorname{aglpD}}-c h i-\mathrm{P}_{y j i Y} t y r A^{\mathrm{fbr}}-\mathrm{P}_{y j i r}$

Str' ${ }^{\mathrm{r}}, \mathrm{pCDM}-\mathrm{P}_{\text {ssrA }}-\mathrm{UTR}_{\mathrm{rpsT}}-\mathrm{CHS}-\mathrm{PUTR} \mathrm{glpD}_{\mathrm{D}}-\mathrm{CHI}$ backbone, $\mathrm{P}_{g l p D}$ and $\mathrm{P}_{y j i \mathrm{Y}}$

pCDM- $\mathrm{P}_{\text {ssra }}-\mathrm{UTR}_{\mathrm{rpsT}}-$ chs-

controlled the expression of $\operatorname{tyr} A^{\mathrm{fbr}}$ and $\operatorname{aro}^{\mathrm{fbr}}$, respectively.

$\mathrm{PUTR}_{\mathrm{glpD}}-$ chi $-\mathrm{P}_{g l p D}-t y r A^{\mathrm{fbr}}-\mathrm{P}_{y j i r}$

Str ${ }^{\mathrm{r}}$, pCDM- $\mathrm{P}_{\text {ssra }}-\mathrm{UTR}_{\mathrm{rps} \mathrm{T}}-\mathrm{CHS}-\mathrm{PUTR}_{\mathrm{glpD}}-\mathrm{CHI}$ backbone, $\mathrm{P}_{g l p D}$ and $\mathrm{P}_{\text {tal }}$

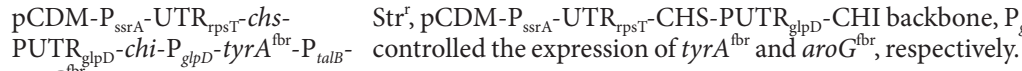

This study

This study

This study

This study

This study

This study

This study

This study

This study

This study aro $G^{\mathrm{fb}}$

J. Microbiol. Biotechnol. 


\section{Fermentation Optimization}

Fermentation seeds were cultured for $12 \mathrm{~h}$ in LB medium and used to optimize the fermentation conditions and medium components. In order to optimize the fermentation temperature, after incubation, the strains were cultured at $37^{\circ} \mathrm{C}$ for different times $(2,4,7$, and $10 \mathrm{~h})$ for cell growth, and then transferred to $30^{\circ} \mathrm{C}, 25^{\circ} \mathrm{C}$, and $20^{\circ} \mathrm{C}$ for $(2 S)$-naringenin production. The titers of (2S)-naringenin and $p$-coumaric acid were measured after $48 \mathrm{~h}$ of fermentation. Furthermore, the initial $\mathrm{pH}$ value (5.0, 6.0, 7.0, and 8.0) was also optimized. All these optimizations were performed in deep 24-well plates. Triplicate biological replicates were performed in every fermentation experiment.

Different concentrations of carbon and nitrogen sources were added in the original MOPS minimal medium in deep 24-well plates to optimize the medium components. Specifically, the optimum concentrations of KAc (1.2, $2.5,5$, and $7.5 \mathrm{~g} / \mathrm{l})$, peptone $(2.5,5,10$, and $15 \mathrm{~g} / \mathrm{l})$, glucose $(5.5,10,15$, and $20 \mathrm{~g} / \mathrm{l})$, ammonia chloride $(6.5,9,14$, and $19 \mathrm{~g} / \mathrm{l})$, yeast extract $(2.5,5,10$, and $15 \mathrm{~g} / \mathrm{l})$, glycerol $(2.5,5,10$, and $15 \mathrm{~g} / \mathrm{l})$, myristic acid $(2.5,5,10$, and $15 \mathrm{~g} / \mathrm{l})$, palmitic acid $(2.5,5,10$, and $15 \mathrm{~g} / \mathrm{l})$, stearic acid $(2.5,5,10$, and $15 \mathrm{~g} / \mathrm{l})$, and cis-11-octadecenoic acid $(2.5,5,10$, and $15 \mathrm{~g} / \mathrm{l})$ in the MOPS minimal medium were investigated. The titers of $(2 S)$-naringenin and $p$-coumaric acid were measured after $48 \mathrm{~h}$ of fermentation in the various modified MOPS media. Then, the components of the final fermentation medium were obtained by a combination of the optimum carbon and nitrogen sources. The fedbatch fermentation was performed in a 5-L bioreactor. Nar-17LM1 strain was used for fed-batch fermentation in the optimum medium at pH 7.0, $1 \mathrm{vvm}$ aeration, and $400 \mathrm{rpm}$ stirring. Then, $100 \mathrm{ml}$ of overnight cultured Nar$17 \mathrm{LM} 1$ was added in $3 \mathrm{~L}$ of fermentation medium. When the $\mathrm{OD}_{600}$ of the cultures reached 3.0 with culture at $37^{\circ} \mathrm{C}$, the temperature was shifted to $25^{\circ} \mathrm{C}$ for (2S)-naringenin production. In this process, $30 \mathrm{ml}$ of $500 \mathrm{~g} / \mathrm{l}$ glucose was added to the bioreactor for $60 \mathrm{~h}, 72 \mathrm{~h}, 90 \mathrm{~h}$, and $96 \mathrm{~h}$ for fermentation.

\section{Plasmid Construction}

The promoters $\mathrm{P}_{\text {talB }}$ and $\mathrm{P}_{g l p D}$ were amplified from the genome of $E$. coli $\mathrm{K} 12 \mathrm{MG} 1655$ by using the primer pairs of $\mathrm{P}_{\text {talB }}$-aroG-F/P $\mathrm{P}_{\text {talB }}$-aroG-R and $\mathrm{P}_{g l p D}-t y r A-\mathrm{F} / \mathrm{P}_{g l p D}-t y r A-\mathrm{R}$, respectively. Promoter $\mathrm{P}_{y j i Y}$ was also amplified from the genome of E. coli K12 MG1655 using the primer pairs of $\mathrm{P}_{y j i Y}$ aroG-F/P $\mathrm{P}_{y j i}$ aroG-R and $\mathrm{P}_{y j i Y} t y r A-\mathrm{F} / \mathrm{P}_{y j i Y} t y r A-\mathrm{R}$. pMD-tyr $A^{\mathrm{fbr}}$-aro $G^{\mathrm{fbr}}$ was linearized by whole plasmid PCR using the primer pairs of tyrA-F/tyrA-R, and then ligated with promoters $\mathrm{P}_{y j i Y}$ and $\mathrm{P}_{g l p D}$ by the Gibson assembly approach to obtain the plasmids $\mathrm{pMD}-\mathrm{P}_{y j i Y}$ tyr $\mathrm{A}^{\mathrm{fbr}}$ aro $G^{\mathrm{fbr}}$ and $\mathrm{pMD}-\mathrm{P}_{g l p D^{-}}-t y r A^{\mathrm{fbr}}$-aro $G^{\mathrm{fbr}}$, respectively. Furthermore, pMD- $\mathrm{P}_{y j i Y}-t y r A^{\mathrm{brr}}$-aro $G^{\mathrm{fbr}}$ and $\mathrm{pMD}-\mathrm{P}_{g l p D^{-}}$ tyr $A^{\mathrm{fbr}}$-aro $G^{\mathrm{fbr}}$ were linearized by whole plasmid PCR using the primer pairs of aroG-F/aroG-R and then ligated with promoters $\mathrm{P}_{y j i Y}$ and $\mathrm{P}_{\text {talB }}$ by the Gibson assembly approach to obtain the plasmids pMD- $\mathrm{P}_{y j i r} t y r A^{\mathrm{fbr}}-\mathrm{P}_{y j i Y}$ aro $G^{\mathrm{fr}}$, pMD- $\mathrm{P}_{y j i Y}-t y r A^{\mathrm{fbr}}-\mathrm{P}_{\text {talB }}$-aro $G^{\mathrm{fbr}}, \mathrm{pMD}-\mathrm{P}_{g l p D}-t y r A^{\mathrm{fbr}}-\mathrm{P}_{y j i Y}$ aro $G^{\mathrm{fbr}}$, and $\mathrm{pMD}-\mathrm{P}_{g l p D}-t y r A^{\mathrm{fbr}}-\mathrm{P}_{\text {talB }}$-aro $G^{\mathrm{fbr}}$. Then, the promoter-tyr $A^{\text {fbr }}$-promoter-aro $G^{\mathrm{fbr}} \mathrm{DNA}$ fragments were amplified from these four plasmids, and ligated with the $\mathrm{XbaI} /$ BamHI digested plasmid pCDM- $\mathrm{P}_{\mathrm{ssr}}-\mathrm{UTR}_{\mathrm{rpsT}}-\mathrm{CHS}-\mathrm{PUTR}_{\mathrm{glpD}}-\mathrm{CHI}$ by the Gibson assembly approach

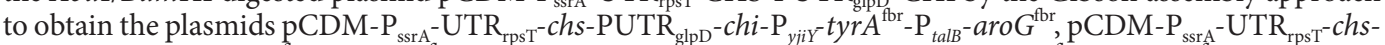

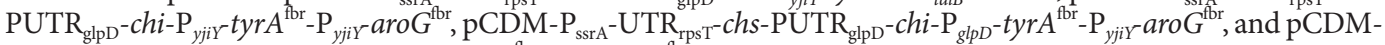

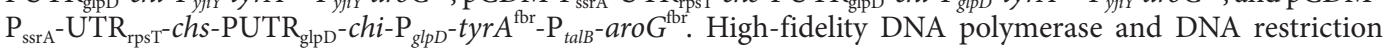
enzymes were purchased from Takara (Dalian, China). The details of the primers used in this study are listed in Table 2.

\section{Analysis Methods}

To quantitatively analyze the titers of (2S)-naringenin and $p$-coumaric acid, we mixed $1 \mathrm{~mL}$ of the fermentation samples with an equivalent volume of $100 \%$ ethyl alcohol and set it at room temperature for $30 \mathrm{~min}$ to lyse the cell wall and dissolve the products. Then, the supernatants of these samples were filtered through $0.22 \mu \mathrm{m}$ nylon 6 filters after 12,000 $\times g$ centrifugation for $5 \mathrm{~min}$. Finally, the filtered supernatants were analyzed by using an Agilent 1100 HPLC system at $290 \mathrm{~nm}$. The degassed mixture of methyl alcohol (41\%), phosphoric acid (0.3\%), and water (58.7\%) was used as the mobile phase after being filtered through $0.22 \mu \mathrm{m}$ nylon 6 filters. A reverse-phase

Table 2. Primers used in this study.

\begin{tabular}{|c|c|}
\hline Primers & Sequence $\left(\text { from } 5^{\prime} \text { to } 3^{\prime}\right)^{*}$ \\
\hline $\mathrm{P}_{y j i y}$-aroG-F & GAAATAATTTTGTTTAACTTTAATAAGGAGATATAGCGGCCGCAAATAACCACTCAGTTATTTACCTTAC \\
\hline $\mathrm{P}_{y j i y}$-aroG-R & CGTAAATCGTCGTTCTGATAATTCATCCCGGGAGTAAAACCTGGCATGTATTGAT \\
\hline $\mathrm{P}_{y j i y}-t y r A-\mathrm{F}$ & TAAAAGCGCGTCGCGGGTAACTGCAGAAATAACCACTCAGTTATTTACCTTAC \\
\hline $\mathrm{P}_{y j i y}-t y r A-\mathrm{R}$ & CGTAATGCGGTCAATTCAGCAACCATGGAGTAAAACCTGGCATGTATTGAT \\
\hline $\mathrm{P}_{\text {talB }}$-aroG-F & GAAATAATTTTGTTTAACTTTAATAAGGAGATATAGCGGCCGCCCTGGCGATAACCGTCTT \\
\hline $\mathrm{P}_{\text {talB }}$-aroG-R & CGTAAATCGTCGTTCTGATAATTCATCCCGGGGATAGTATTTCTCTTTAAACAGCTTGT \\
\hline $\mathrm{P}_{g l p D}-\mathrm{t} y r A-\mathrm{F}$ & TAAAAGCGCGTCGCGGGTAACTGCAGTCACTCTAAAATGTTTTTTCAATGT \\
\hline $\mathrm{P}_{g l p D}-t y r A-\mathrm{R}$ & CGTAATGCGGTCAATTCAGCAACCATGGGCTGCCCTCATTCACTTTC \\
\hline $\operatorname{aroG}-\mathrm{F}$ & CAATTCCCCTGTAGAAATAATTTTGTTTAAC \\
\hline aroG-R & GTAACTCTTTGATTTCTTTGATGCGTAAATC \\
\hline tyrA-F & CTGTTACGTCAACTGGCGAATGC \\
\hline tyrA-R & TATCGACTTCATCAATTTGATCGCG \\
\hline tyrA-aroG-F & TGATCTTTTCTACTGAACCGCTCTAGACCCCTGTAGAAATAATTTTGTTTAACTTTAATA \\
\hline tyrA-aroG-R & CGGATGTGATAGCCAATGGATCCGACCATGATTACGCCAAGTTTGC \\
\hline
\end{tabular}


C18 column $(4.6 \times 150 \mathrm{~mm}$, Thermo, USA $)$ was used to separate $(2 S)$-naringenin and $p$-coumaric acid at $35^{\circ} \mathrm{C}$ with a constant flow rate of $1 \mathrm{ml} / \mathrm{min}$.

\section{Results}

\section{Optimization of Medium Components}

Medium components not only influence cell growth, they are also closely related to the production of (2S)naringenin. To supply optimal growth and production conditions, we first optimized the concentration of different carbon sources, nitrogen sources, fatty acids, and $\mathrm{Mn}^{2+}$. In doing so, Mut-17 was used as the producer, and MOPS minimal medium was used as the initial fermentation medium. Because barren MOPS medium is not suited to cell growth, we optimized the carbon sources and nitrogen sources. The results show that yeast extract and peptone significantly improved the growth state of Mut-17, but negatively impacted (2S)-naringenin production (Fig. 2). With increasing concentrations of yeast extract and peptone, the titer of (2S)-naringenin gradually decreased. However, the titer of $p$-coumaric acid gradually decreased and increased with yeast extract and peptone, respectively. Furthermore, high concentrations of the inorganic nitrogen source $\mathrm{NH}_{4} \mathrm{Cl}$ significantly repressed cell growth and $(2 \mathrm{~S})$-naringenin production. Hence, $4 \mathrm{~g} / \mathrm{l} \mathrm{NH}_{4} \mathrm{Cl}$ was the optimum nitrogen source.

Malonyl-CoA is the main limiting factor for $(2 S)$-naringenin biosynthesis $[21,22]$. Hence, it is necessary to enhance the production of malonyl-CoA at the fermentation level. The direct precursor of malonyl-CoA is acetylCoA, whose biosynthesis is closely related to carbon sources and acetate (Fig. 1). With this background, we optimized the concentrations of glucose, glycerol, and potassium acetate (KAc) in the MOPS medium. We found that a high glucose concentration significantly improved the titer of $(2 S)$-naringenin, and the highest titer reached 2.96-fold that of the control when $20 \mathrm{~g} / \mathrm{l}$ glucose was added (Fig. 2). However, $15 \mathrm{~g} / \mathrm{l}$ glucose almost achieved the highest titer and had the highest final $\mathrm{OD}_{600}$. Hence, we used $15 \mathrm{~g} / \mathrm{l}$ glucose as the optimum concentration. On the other hand, both glycerol and KAc were beneficial for (2S)-naringenin production and cell growth. Different glycerol concentrations had no significant influence on $(2 S)$-naringenin biosynthesis and cell growth, but higher concentrations of KAc improved them. Overall, the optimum carbon sources were glucose at $15 \mathrm{~g} / \mathrm{l}$, glycerol at $2.5 \mathrm{~g} / \mathrm{l}$, and $\mathrm{KAc}$ at $7.5 \mathrm{~g} / \mathrm{l}$.

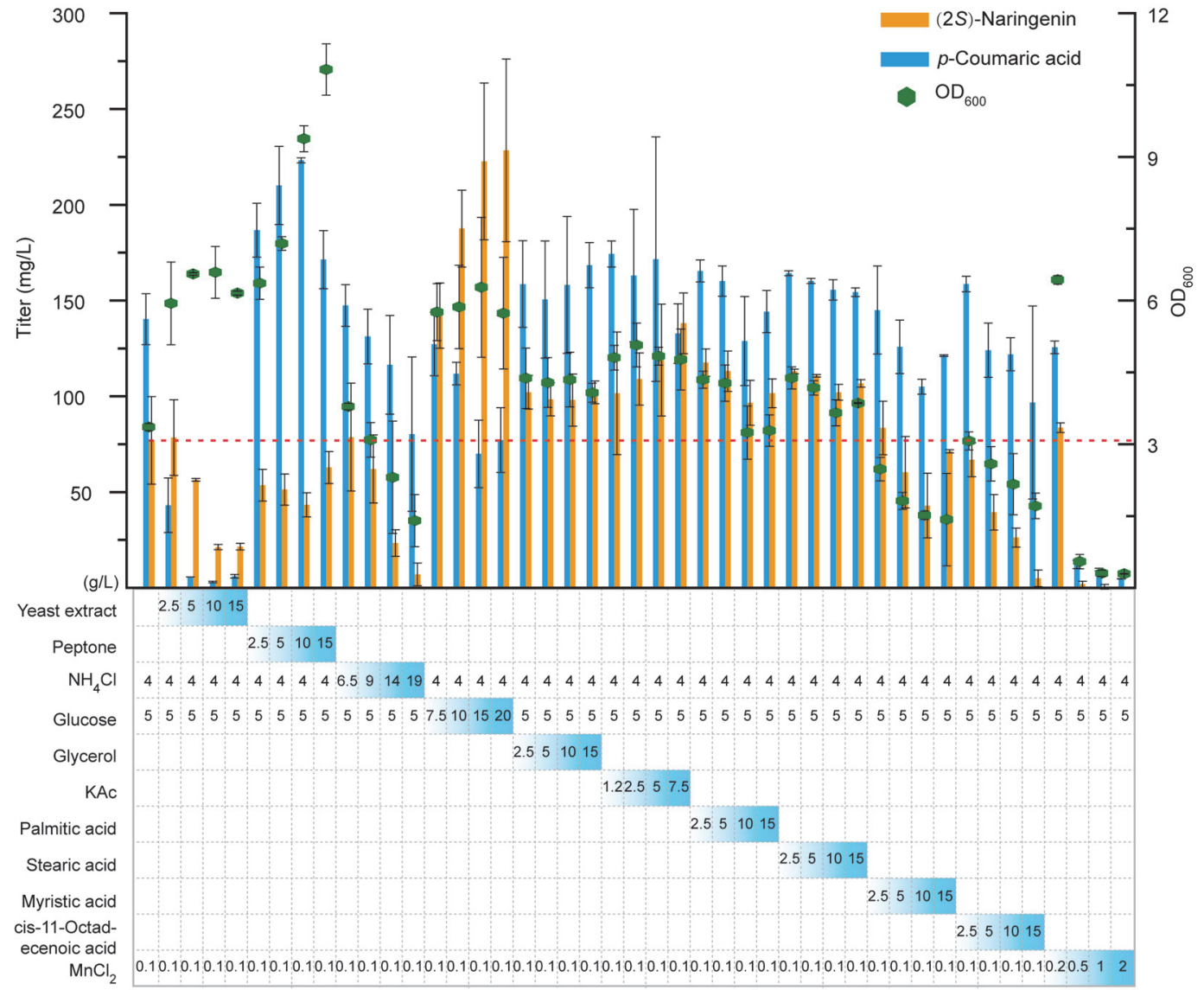

Fig. 2. The influence of medium components on growth state, as well as (2S)-naringenin and $p$-coumaric acid titer, of Mut-17. Different concentrations of extra nitrogen sources, carbon sources, fatty acids, and $\mathrm{MnCl}_{2}$ were added to the MOPS minimal medium. MOPS minimal medium already contains $4 \mathrm{~g} / \mathrm{NH}_{4} \mathrm{Cl}, 5 \mathrm{~g} / \mathrm{l}$ glucose, and $0.1 \mathrm{~g} / \mathrm{l} \mathrm{MnCl}$. Fermentation in MOPS minimal medium was used as the control. The red dashed line represents the (2S)-naringenin titer of the control. 
Malonyl-CoA mainly flows into the native fatty acid pathway. Thus, repressing the metabolic flux of the fatty acid pathway could efficiently enhance the accumulation of malonyl-CoA [23]. Here, the dynamic regulation system of Mut-17 could repress the fatty acid synthesis pathway according to the concentration of (2S)-naringenin to enhance the accumulation of malonyl-CoA. Hence, we speculated that adding extra fatty acid would improve cell growth and (2S)-naringenin production. To prove this, we added different concentrations of palmitic acid, stearic acid, myristic acid, and cis-11-octadecenoic acid into the MOPS medium for fermentation. The results show that $2.5 \mathrm{~g} / \mathrm{l}$ each of palmitic acid and stearic acid was the optimum concentration for both cell growth and $(2 S)$-naringenin production (Fig. 2). Adding myristic acid and cis-11-octadecenoic acid repressed both cell growth and (2S)-naringenin production. On the other hand, acyl carrier protein (ACP) is an important protein in the fatty acid biosynthesis process. Repressing the activity of ACP improved the accumulation of malonyl-CoA. Given that $\mathrm{AcpH}$ is an $\mathrm{Mn}^{2+}$-dependent phosphodiesterase [24], optimizing the concentration of $\mathrm{Mn}^{2+}$ would probably be helpful for fermentation. For this reason, we added different concentrations of $\mathrm{MnCl}_{2}$ into the MOPS medium and found that high concentrations significantly repressed cell growth (Fig. 2). Finally, the optimum concentration of $\mathrm{MnCl}_{2}$ was identified as $0.1 \mathrm{~g} / \mathrm{l}$.

\section{Optimization of the Fermentation Conditions}

Temperature and $\mathrm{pH}$ are important factors that can significantly influence cell growth and pathway enzyme activity. Generally, fermentation requires a set of conditions for cell growth in the early growth stage and then a set of conditions for the production stage. Wherein, temperature not only influenced cell growth but also significantly influenced the activity of enzymes. Furthermore, the enzymes from different origins usually have different optimum catalyzation temperature [25]. With this background, we investigated a temperature-shift fermentation strategy. Mut- 17 was cultured at $37^{\circ} \mathrm{C}$ for different times for cell growth, and then transferred to a lower temperature for $(2 \mathrm{~S})$-naringenin biosynthesis (Fig. 3A). With increasing culture times at $37^{\circ} \mathrm{C}$, the titers of $(2 S)$-naringenin and $p$-coumaric acid were significantly decreased. A low temperature in the production stage promoted $(2 S)$-naringenin biosynthesis, but reduced the biosynthesis of $p$-coumaric acid (Fig. $3 \mathrm{~A}$ ). This indicates that the optimum catalyzation temperature of TAL was probably different with the downstream enzymes (4CL, $\mathrm{CHS}$, and $\mathrm{CHI}$ ). Wherein, the activity of downstream enzymes would increase under lower temperature and thus enhanced the conversion from $p$-coumaric acid to $(2 S)$-naringenin. Finally, we identified that culturing at $37^{\circ} \mathrm{C}$ for $2 \mathrm{~h}$ and then transferring to $25^{\circ} \mathrm{C}$ was the optimum temperature-shift fermentation condition. In addition, $\mathrm{pH}$ can also influence cell growth and production. Hence, we optimized the initial $\mathrm{pH}$. The results show that a neutral or alkaline $\mathrm{pH}$ condition was better than a faintly acid condition (Fig. 3B). The optimum initial pH was identified as 7.0.

Fermentation was subsequently performed in a shake flask under the above optimum medium and conditions (that is, adding $4 \mathrm{~g} / \mathrm{l} \mathrm{NH}_{4} \mathrm{Cl}, 15 \mathrm{~g} / \mathrm{l}$ glucose, $2.5 \mathrm{~g} / \mathrm{l}$ glycerol, $7.5 \mathrm{~g} / \mathrm{l} \mathrm{KAc}, 2.5 \mathrm{~g} / \mathrm{l}$ palmitic acid, and $2.5 \mathrm{~g} / \mathrm{l}$ stearic acid in MOPS minimal medium, initial pH $7.0,37^{\circ} \mathrm{C}$ culture for $2 \mathrm{~h}$ and then transfer to $25^{\circ} \mathrm{C}$ ). To maintain a pH higher than 5.6 in the fermentation process, we added $5 \mathrm{~g} / \mathrm{l} \mathrm{CaCO}_{3}$ to the shake flasks. Finally, Mut- 17 produced $391 \mathrm{mg} / \mathrm{l}$ (2S)-naringenin from $3 \mathrm{mM}$ L-tyrosine with $24 \mathrm{mg} / \mathrm{l}$ p-coumaric acid accumulation (Fig. $4 \mathrm{~A}$ ). This (2S)-naringenin titer was 1.6-fold (391 mg/l vs. $251 \mathrm{mg} / \mathrm{l}$ ) higher than the un-optimized titer in our previous report [19].

\section{Releasing the Feedback Inhibition for De Novo Synthesis of (2S)-Naringenin}

In the tyrosine synthesis pathway, aroG and tyrA could be feedback-repressed by tyrosine. Hence, we overexpressed their anti-feedback inhibition mutants ( $\operatorname{aro} G^{\mathrm{fbr}}$ and $\left.t y r A^{\mathrm{fbr}}\right)$ [14] in Mut-17. Three different strength promoters $-\mathrm{P}_{y j i Y}$ (low strength), $\mathrm{P}_{\text {talB }}$ (medium strength), and $\mathrm{P}_{g l p D}$ (high strength) [26]-were used to control the
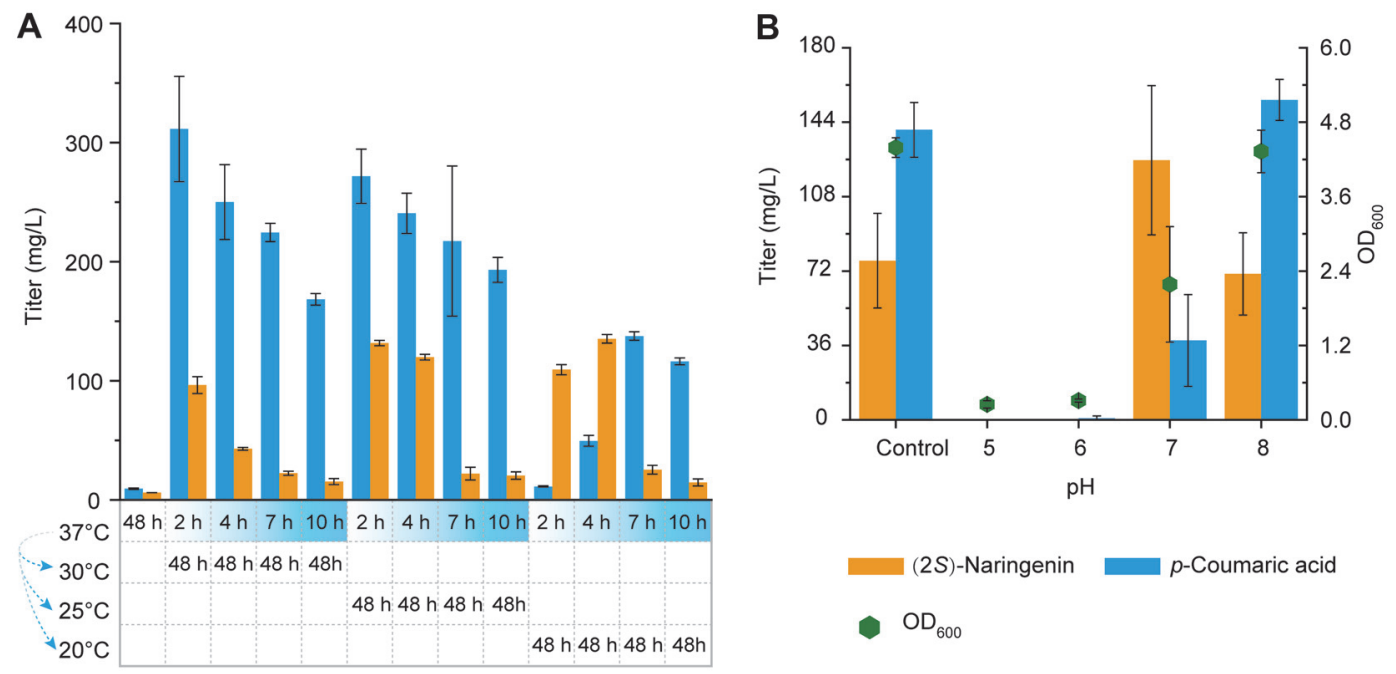

Fig. 3. Temperature and $\mathbf{p H}$ optimization. (A) Temperature-shift strategy to optimize the time and temperature of the growth stage and production stage. (B) Initial $\mathrm{pH}$ optimization. The control had a natural $\mathrm{pH}$ (7.4) of MOPS. 
A

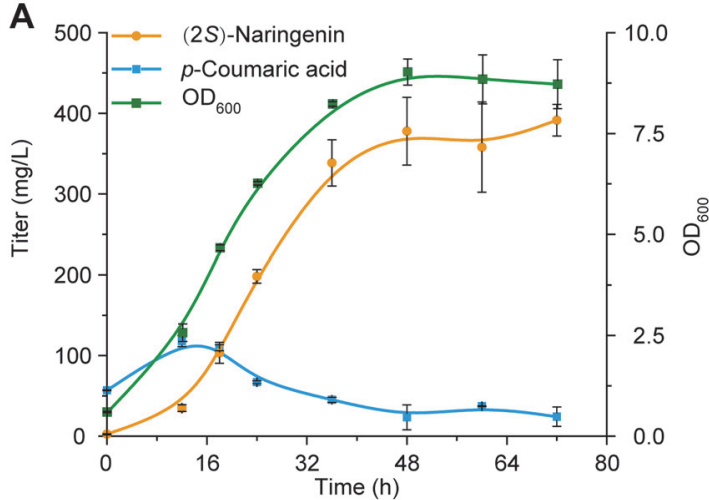

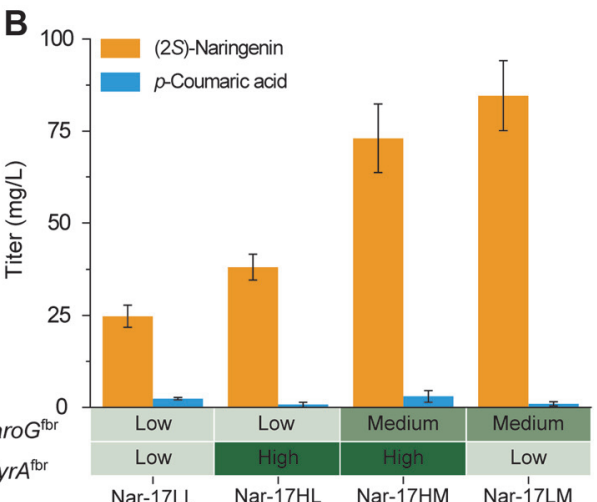

Fig. 4. Shake flask fermentation in the optimum medium for (2S)-naringenin production from tyrosine (A) and glucose (B). (A) Mut-17 was used for fermentation; (B) The expression levels of the anti-feedback inhibition genes $\left(\operatorname{aro} G^{\mathrm{br}}\right.$ and $t y r A^{\mathrm{fbr}}$ ) were optimized by promoters with different strengths. $0 \mathrm{~h}$ represents the starting time of temperature shifting.

expression levels of aro $G^{\mathrm{fbr}}$ and $t y r A^{\mathrm{fbr}}$. Comparing Nar-17LL with Nar-17-LM and Nar-17HL with Nar-17-HM, we found that the higher expression level of aro $G^{\mathrm{br} r}$ yielded a higher (2S)-naringenin titer (Fig. 4B). Hence, aro $G^{\text {fbr }}$ was probably the rate-limiting step for the biosynthesis of tyrosine. The highest titer of (2S)-naringenin was 85 $\mathrm{mg} / \mathrm{l}$, which was far lower than the titer in Mut-17. We also analyzed the accumulation level of $p$-coumaric acid and tyrosine and found that the accumulation of $p$-coumaric acid was lower than $3 \mathrm{mg} / \mathrm{l}$, and no tyrosine was detected. Such results indicate that the current metabolic flux of the tyrosine biosynthesis pathway could not satisfy the demands of downstream (2S)-naringenin biosynthesis.

\section{Enhancing the Metabolic Flux from Glucose to L-Tyrosine}

In order to further improve the metabolic flux of the tyrosine biosynthesis pathway, we introduced the (2S)naringenin synthesis pathway (including pETM-PUTR trxa $-T_{A L}-\mathrm{PUTR} \mathrm{talB}_{\mathrm{ta}}-4 \mathrm{CL}, \mathrm{pCDM}-\mathrm{P}_{\mathrm{ssrA}}-\mathrm{UTR}_{\mathrm{rpsT}}-$ chs-

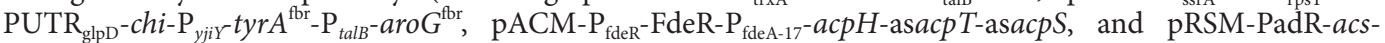
ACC, see Table 1) into four knockout strains [27] and generated strains of Nar-17LM1, Nar-17LM2, Nar-17LM3, and Nar-17LM4 (Fig. 5A). TyrR is a tyrosine-activated repressor that can repress the expression of aroH, aroF, and tyrB (Fig. 1). After knocking out tyrR, the titer of (2S)-naringenin improved to $485 \mathrm{mg} / \mathrm{l}$, which was 5.7 -fold higher than that of Nar-17-LM. The second knockout target was the phosphotransferase system (PTS) since the function of PTS needs to sacrifice the accumulation of PEP, which is a precursor of tyrosine [28]. ptsG and crr are the two components of PTS. However, after their deletion, the titer of (2S)-naringenin decreased to $137 \mathrm{mg} / \mathrm{l}$, and the titer of $p$-coumaric acid and tyrosine increased to $558 \mathrm{mg} / \mathrm{l}$ and $590 \mathrm{mg} / \mathrm{l}$, respectively (Fig. 5A). Such results indicate that too much tyrosine accumulation probably repressed the production of $(2 S)$-naringenin for unknown reasons. In previous study, $\mathrm{Gu}$ et al. showed that the knockout of $p y k F$ could significantly enhance the metabolic flux of

A

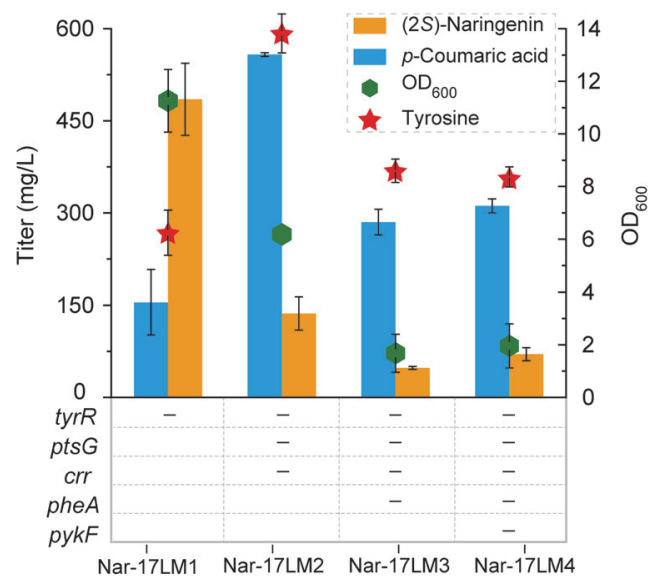

B

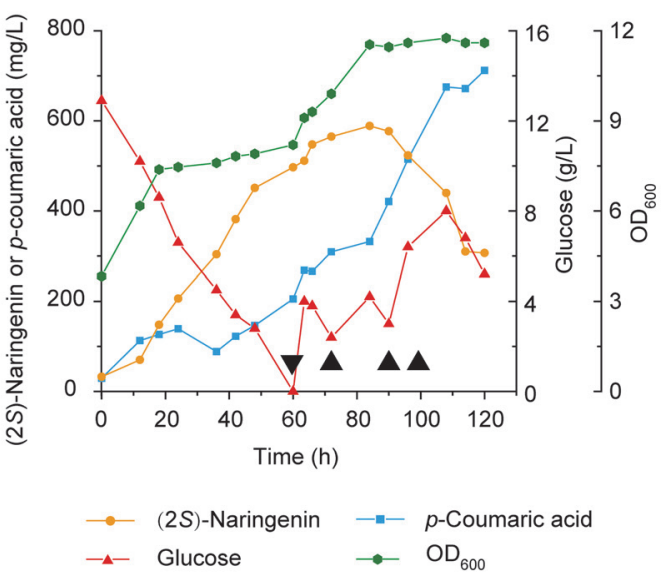

Fig. 5. De novo biosynthesis of (2S)-naringenin by knockout strains in a shake flask (A) and 5-L bioreactor (B). (A) A minus represents the relative genes that were knocked out; (B) Nar-17LM1 was the producer for fed-batch fermentation. Black triangles represent the time points at which $30 \mathrm{~mL}$ of $500 \mathrm{~g} / \mathrm{l}$ glucose was fed. $0 \mathrm{~h}$ represents the starting time of temperature shifting. 
tyrosine biosynthesis [29]. However, in this study, knocking out pheA and $p y k F$ significantly influenced cell growth, thus reducing $(2 S)$-naringenin production.

\section{Fed-Batch Fermentation in a 5-L Bioreactor}

To further improve the titer of (2S)-naringenin, the best producer Nar-17LM1 was used for fed-batch fermentation in a 5-L bioreactor. The $\mathrm{pH}$ was maintained at 7.0 throughout the fermentation process. We found that the glucose was depleted after $60 \mathrm{~h}$ fermentation at $25^{\circ} \mathrm{C}$. Feeding extra glucose improved the titer of $(2 S)$ naringenin, achieving the highest (2S)-naringenin production of $588 \mathrm{mg} / \mathrm{l}$ (Fig. 5B). However, (2S)-naringenin started to degrade after $84 \mathrm{~h}$ fermentation, and the $p$-coumaric acid accumulation started rapidly. This phenomenon probably represents the $(2 S)$-naringenin consumption pathway that exists naturally in E. coli.

\section{Discussion}

(2S)-Naringenin biosynthesis in E. coli has suffered from low production. The reported highest titer was $100.64 \mathrm{mg} / \mathrm{l}$ with glucose as the substrate [14]. Furthermore, an expensive inducer (isopropyl- $\beta$-d-thiogalactoside) was used in the fermentation process, which elevated the biosynthesis cost. To overcome these challenges, using our previously constructed dynamic regulation strain (Mut-17) [19], we systemically optimized the components of the fermentation medium and fermentation conditions, obtaining $391 \mathrm{mg} / \mathrm{l}(2 \mathrm{~S})$-naringenin from $3 \mathrm{mM} \mathrm{L}$ tyrosine. Furthermore, we released the tyrosine feedback inhibition by deleting $\operatorname{tyr} R$ and overexpressing aro $G^{\mathrm{fbr}}$ and $t y r A^{\text {fbr }}$. The optimum strain Nar-17LM1 produced $588 \mathrm{mg} / \mathrm{l}$ of $(2 S)$-naringenin from glucose, the highest titer in E. coli. Compared with S. cerevisiae and Yarrowia lipolytica, the other two commonly used hosts, E. coli exhibited a shorter fermentation process. The reported highest de novo production titer of $(2 S)$-naringenin in S. cerevisiae and Y. lipolytica was $220 \mathrm{mg} / \mathrm{l}$ [30] and $898 \mathrm{mg} / \mathrm{l}$ [18], respectively. Although the (2S)-naringenin titer of Nar-17LM1 was lower than Y. lipolytica, the fermentation process was significantly shorter ( $84 \mathrm{~h} \mathrm{vs.} 300 \mathrm{~h}$ ), making Nar-17LM1 a suitable producer for (2S)-naringenin.

The de novo biosynthesis pathway of (2S)-naringenin usually expresses more than 10 genes. Incongruous expression of these genes in one host often results in low productivity or impaired cell growth [31]. Hence, coculture strategies were established in recent years to split the long pathways into different strains for reducing cell burden. To do so, Zhang et al. split the (2S)-naringenin de novo synthesis pathway into two parts: tyrosine synthesis pathway that uses $\mathrm{D}$-xylose as the substrate, and $(2 S)$-naringenin synthesis pathway that uses tyrosine as the substrate [32]. Tyrosine and (2S)-naringenin synthesis pathways were overexpressed in E. coli and S. cerevisiae, respectively. After a series of optimizations, they obtained $21.16 \mathrm{mg} / \mathrm{l}(2 \mathrm{~S})$-naringenin. Likewise, Ganesan et al. used a $p$-coumaric acid-producing strain and a (2S)-naringenin-producing strain in a co-culture strategy [33] and obtained $40 \mathrm{mg} / \mathrm{l}(2 \mathrm{~S})$-naringenin. However, it is difficult to fine-tune a co-culture system in the fermentation process, leading to low productivity. The transportation of intermediates, such as tyrosine and $p$-coumaric acid, further reduces the synthesis efficiency since such intermediates need to transfer to other cells for (2S)-naringenin biosynthesis. Here, we used a dynamic regulated strain as a platform for de novo production of (2S)-naringenin. This strain is capable of automatically regulating its metabolic flux to balance cell growth and (2S)-naringenin production, and thus, reduce the burden caused by the expression of a long metabolic pathway. As the point of comparison with other dynamic regulated strains, the $(2 S)$-naringenin titer of Nar-17LM1 was 3.7- and 4.3-fold of the reports of Lv et al. [34] and Dinh et al. [35, 36], respectively.

In the fermentation process, we found that organic nitrogen sources, such as yeast extract and peptone, promoted cell growth but reduced (2S)-naringenin production (Fig. 2). One possible reason was that too fast cell growth would exhaust the cell resources that should be used to express the biosynthesis pathway of (2S)naringenin. Hence, a more flexible strategy needs to be developed to distribute cell resources between cell growth and $(2 S)$-naringenin production. On the other hand, the fermentation temperature significantly influenced the production of $(2 S)$-naringenin and $p$-coumaric acid. A higher temperature $\left(30^{\circ} \mathrm{C}\right)$ was suitable for $p$-coumaric acid production, but a lower temperature $\left(20^{\circ} \mathrm{C}\right)$ was suitable for $(2 S)$-naringenin production (Fig. 3A). This phenomenon was probably caused by differences in the optimum catalyzation temperature between TAL and the downstream enzymes (4CL, CHS, and CHI). In this regard, future research is needed for screening or engineering TAL to fit the catalyzation temperature of the downstream enzymes. Taken together, the results presented in this study indicate that $E$. coli is still a superior host for producing $(2 S)$-naringenin or other flavonoids.

\section{Acknowledgments}

This work was supported by the National Science Fund for Excellent Young Scholars (21822806), the National Natural Science Foundation of China (31900066 and 31770097), and the Fundamental Research Funds for the Central Universities (JUSRP12056).

\section{Conflict of Interest}

The authors have no financial conflicts of interest to declare.

\section{References}

1. Orhan IE, Nabavi SF, Daglia M, Tenore GC, Mansouri K, Nabavi SM. 2015. Naringenin and atherosclerosis: a review of literature. Curr. Pharm. Biotechnol. 16: 245-251.

2. Liu HL, Jiang WB, Xie MX. 2010. Flavonoids: recent advances as anticancer drugs. Recent Pat. Anticancer Drug Discov. 5: 152-164.

3. Skarpalezos D, Detsi A. 2019. Deep eutectic solvents as extraction media for valuable flavonoids from natural sources. Appl. Sci. 9: 11749-11752. 
4. Sharma K, Mahato N, Lee YR. 2019. Extraction, characterization and biological activity of citrus flavonoids. Rev. Chem. Eng. 35: 265284.

5. Guo P, Yan W, Han Q, Wang C, Zhang Z. 2015. Simultaneous quantification of 25 active constituents in the total flavonoids extract from Herba Desmodii Styracifolii by high-performance liquid chromatography with electrospray ionization tandem mass spectrometry. J. Sep. Sci. 38: 1156-1163.

6. Wu JJ, Du GC, Zhou JW, Chen J. 2014. Systems metabolic engineering of microorganisms to achieve large-scale production of flavonoid scaffolds. J. Biotechnol. 188: 72-80.

7. Zhou S, Lyu Y, Li H, Koffas MA, Zhou J. 2019. Fine-tuning the (2S)-naringenin synthetic pathway using an iterative high-throughput balancing strategy. Biotechnol. Bioeng. 116: 1392-1404.

8. Salehi B, Fokou PVT, Sharifi-Rad M, Zucca P, Pezzani R, Martins N, et al. 2019. The therapeutic potential of naringenin: a review of clinical trials. Pharmaceuticals 12: 11.

9. Wu JJ, Yu O, Du GC, Zhou JW, Chen J. 2014. Fine-tuning of the fatty acid pathway by synthetic antisense RNA for enhanced (2S)naringenin production from L-tyrosine in Escherichia coli. Appl. Environ. Microbiol. 80: 7283-7292.

10. Wu JJ, Du GC, Chen J, Zhou JW. 2015. Enhancing flavonoid production by systematically tuning the central metabolic pathways based on a CRISPR interference system in Escherichia coli. Sci. Rep-UK 5: 13477.

11. Lyu X, Ng KR, Lee JL, Mark R, Chen WN. 2017. Enhancement of naringenin biosynthesis from tyrosine by metabolic engineering of Saccharomyces cerevisiae. J. Agr. Food Chem. 65: 6638-6646.

12. Gao S, Lyu Y, Zeng W, Du G, Zhou J, Chen J. 2020. Efficient biosynthesis of (2S)-naringenin from p-coumaric acid in Saccharomyces cerevisiae. J. Agric. Food Chem. 68: 1015-1021.

13. Gao S, Zhou H, Zhou J, Chen J. 2020. Promoter library based pathway optimization for efficient (2S)-naringenin production from $p$ coumaric acid in Saccharomyces cerevisiae. J. Agr. Food Chem. 68: 6884-6891.

14. Wu JJ, Zhou TT, Du GC, Zhou JW, Chen J. 2014. Modular optimization of heterologous pathways for de novo synthesis of (2S)naringenin in Escherichia coli. PLoS One 9: e101492.

15. Raman S, Rogers JK, Taylor ND, Church GM. 2014. Evolution-guided optimization of biosynthetic pathways. Proc. Natl. Acad. Sci. USA 111: 17803-17808

16. Lv Y, Marsafari M, Koffas M, Zhou J, Xu P. 2019. Optimizing oleaginous yeast cell factories for flavonoids and hydroxylated flavonoids biosynthesis. ACS Synth. Biol. 8: 2514-2523.

17. Wei W, Zhang P, Shang Y, Zhou Y, Ye B-C. 2020. Metabolically engineering of Yarrowia lipolytica for the biosynthesis of naringenin from a mixture of glucose and xylose. Bioresour. Technol. 314: 123726.

18. Palmer CM, Miller KK, Nguyen A, Alper HS. 2020. Engineering 4-coumaroyl-CoA derived polyketide production in Yarrowia lipolytica through a $\beta$-oxidation mediated strategy. Metab. Eng. 57: 174-181.

19. Zhou S, Yuan S-F, Nair PH, Alper HS, Deng Y, Zhou J. 2020. Development of a growth coupled dynamic regulation network balancing malonyl-CoA node to enhance (2S)-naringenin synthesis in E. coli. BioRxiv 7: 192633.

20. Neidhardt FC, Bloch PL, Smith DF. 1974. Culture medium for enterobacteria. J. Bacteriol. 119: 736-747.

21. Pandey RP, Parajuli P, Koffas MAG, Sohng JK. 2016. Microbial production of natural and non-natural flavonoids: pathway engineering, directed evolution and systems/synthetic biology. Biotechnol. Adv. 34: 634-662.

22. Zhou S, Hao T, Xu S, Deng Y. 2020. Coenzyme A thioester-mediated carbon chain elongation as a paintbrush to draw colorful chemical compounds. Biotechnol. Adv. 43: 107575

23. Xu P, Ranganathan S, Fowler ZL, Maranas CD, Koffas MAG. 2011. Genome-scale metabolic network modeling results in minimal interventions that cooperatively force carbon flux towards malonyl-CoA. Metab. Eng. 13: 578-587.

24. Heil CS, Wehrheim SS, Paithankar KS, Grininger M. 2019. Fatty acid biosynthesis: chain-length regulation and control. ChemBioChem. 20: 2298-2321.

25. Arcus VL, Prentice EJ, Hobbs JK, Mulholland AJ, Van der Kamp MW, Pudney CR, et al. 2016. On the temperature dependence of enzyme-catalyzed rates. Biochemistry 55: 1681-1688.

26. Zhou S, Ding R, Chen J, Du G, Li HZ, Zhou J. 2017. Obtaining a panel of cascade promoter-5'-UTR complexes in Escherichia coli. ACS Synth. Biol. 6: 1065-1075.

27. Fordjour E, Adipah FK, Zhou S, Du G, Zhou J. 2019. Metabolic engineering of Escherichia coli BL21 (DE3) for de novo production of L-DOPA from D-glucose. Microb. Cell Fact. 18: 74.

28. Deutscher J, Aké FMD, Derkaoui M, Zébré AC, Cao TN, Bouraoui H, et al. 2014. The bacterial phosphoenolpyruvate: carbohydrate phosphotransferase system: regulation by protein phosphorylation and phosphorylation-dependent protein-protein interactions. Microbiol. Mol. Biol. Rev. 78: 231-256.

29. Gu Y, Ma J, Zhu Y, Ding X, Xu P. 2020. Engineering Yarrowia lipolytica as a chassis for de novo synthesis of five aromatic-derived natural products and chemicals. ACS Synth. Biol. 9: 20936.

30. Lyu X, Zhao G, Ng KR, Mark R, Chen WN. 2019. Metabolic engineering of Saccharomyces cerevisiae for de novo production of Kaempferol. J. Agric. Food Chem. 67: 5596-5606.

31. Jones JA, Vernacchio VR, Sinkoe AL, Collins SM, Ibrahim MHA, Lachance DM, et al. 2016. Experimental and computational optimization of an Escherichia coli co-culture for the efficient production of flavonoids. Metab. Eng. 35: 55-63.

32. Zhang W, Liu H, Li X, Liu D, Dong XT, Li FF, et al. 2017. Production of naringenin from D-xylose with co-culture of $E$. coli and S. cerevisiae. Eng. Life Sci. 17: 1021-1029.

33. Ganesan V, Li Z, Wang X, Zhang H. 2017. Heterologous biosynthesis of natural product naringenin by co-culture engineering. Synth. Syst. Biotechnol. 2: 236-242.

34. Lv Y, Gu Y, Xu J, Zhou J, Xu P. 2020. Coupling metabolic addiction with negative autoregulation to improve strain stability and pathway yield. Metab. Eng. 61: 79-88.

35. Dinh CV, Chen X, Prather KLJ. 2020. Development of a quorum-sensing based circuit for control of coculture population composition in a naringenin production system. ACS Synth. Biol. 9: 590-597.

36. Dinh CV, Prather KL. 2019. Development of an autonomous and bifunctional quorum-sensing circuit for metabolic flux control in engineered Escherichia coli. Proc. Natl. Acad. Sci. USA 116: 25562-25568.

37. Siedler S, Stahlhut SG, Malla S, Maury J, Neves AR. 2014. Novel biosensors based on flavonoid-responsive transcriptional regulators introduced into Escherichia coli. Metab. Eng. 21: 2-8.

38. Siedler S, Khatri NK, Zsohár A, Kjærbølling I, Vogt M, Hammar P, et al. 2017. Development of a bacterial biosensor for rapid screening of yeast p-coumaric acid production. ACS Synth. Biol. 6: 1860-1869. 